\title{
Mortes súbitas em bovinos causadas pela ingestão de Mascagnia sp (Malpighiaceae), no Estado de Santa Catarina ${ }^{1}$
}

\author{
Aldo Gava², José Cristani ${ }^{3}$, José V. Branco ${ }^{4}$, Dalmo S. Neves ${ }^{2}$, Ademir J. Mondadori² \\ e Renato S. Sousa ${ }^{5}$
}

\begin{abstract}
Gava A., Cristani J., Branco J. V., Neves D.S., Mondadori A.J. \& Sousa R.S. 1998. [Sudden death in cattle by Mascagnia sp (Malpighiaceae) in the State of Santa Catarina, Brazil.] Mortes súbitas em bovinos causadas pela ingestão de Mascagnia sp (Malpighiaceae), no Estado de Santa Catarina. Pesquisa Veterinária Brasileira 18(1):16-20. Laboratório de Patologia Animal, Centro de Ciências Agroveterinárias, UDESC, Av. Luiz de Camões 2090, Lages, SC 88520-000, Brazil.

In the coastal areas of the State of Santa Catarina occurs a disease in cattle characterized by "sudden death". The disease was reproduced in bovines by oral administration of Mascagnia sp. Single doses of $5 \mathrm{~g} / \mathrm{kg}$ of the fresch leaves of Mascagnia sp caused non-lethal poisoning; single doses of 7,5 g/kg caused lethal poisoning in one of two bovines and doses of $10 \mathrm{~g} / \mathrm{kg}$ death in two others. In the experiments clinical manifestations were observed when the animals were exercised. They consisted in weakness, swollen jugular vein, slight muscular tremors and sometimes sudden contractions; tachycardia, already observed before the exercise, became more intense. At the end the animals suddenly laid down or fell on their side. The course of poisoning in those two animals which were followed up till death, was 40 and 75 minutes. The highest doses given $(15$ and $20 \mathrm{~g} / \mathrm{kg}$ ) caused a protratecd course; the animals got slow and apathic, avoiding any movements. They were found dead, $7 \mathrm{~h} 45 \mathrm{~min}$ and 21 hours after the first clinical signs. These two last experiments show the importance of exercise regarding the onset of symptoms and the occurrence of "sudden death". The main postmortem findings were intense red discoloration of the mucosa of the small intestine and oedema of the gallbladder wall. The main histological alteration was hydropic vacuolar degeneration of the tubular epithelium of the kidney in three of the five bovines which died.
\end{abstract}

INDEX TERMS: Poisonous plants, plant poisoning, Mascagnia sp, cattle, pathology, "sudden death".

RESUMO.- No Litoral de Santa Catarina vem ocorrendo uma doença de bovinos caracterizada por "morte súbita". Para esclarecer a etiologia, foram conduzidos experimentos em bovinos nos quais se reproduziu a enfermidade pela administração oral de Mascagnia sp. Doses únicas de $5 \mathrm{~g} / \mathrm{kg}$ das folhas frescas de Mascagnia sp causaram intoxicação não letal. Doses únicas de 7,5 g/kg causaram intoxicação letal em um de dois bovinos e dose de $10 \mathrm{~g} / \mathrm{kg}$ a morte de outros dois.

\footnotetext{
${ }^{1}$ Aceito para publicação em 16 de setembro de 1997.

${ }^{2}$ Departamento de Clínica e Patologia, Centro de Ciências Agroveterinárias (CAV), Universidade do Estado de Santa Catarina (UDESC), Av.Luiz de Camões 2090, Lages, SC 88520-000.

${ }^{3}$ Departamento de Zootecnia, CAV, UDESC.

${ }^{4}$ Veterinário da Cooperativa de Tubarão, SC.

${ }^{5}$ Mestrando em Patologia Veterinária, UFSM, RS.
}

Nessas dosagens de 5 a $10 \mathrm{~g} / \mathrm{kg}$ as manifestações clínicas eram observadas quando os animais eram movimentados, e consistiram em cansaço, jugular ingurgitada, leves tremores musculares e às vezes contrações bruscas; taquicardia já notada antes do exercício se acentuava. Finalmente os animais se deitavam ou caíram subitamente. A evolução da intoxicação nos dois animais em que foi acompanhada até a morte, foi de 40 e 75 minutos. As mais altas doses administradas (15 e $20 \mathrm{~g} / \mathrm{kg}$ ) provocaram um quadro de intoxicação protraída; os animais se mostraram lerdos e apáticos, evitando quaisquer movimentos; foram encontrados mortos $7 \mathrm{~h} 45 \mathrm{~min}$ e 21 horas após terem sido observados os primeiros sinais clínicos. Esses dois últimos experimentos mostram que a movimentação é um fator importante para a manifestação da "morte súbita”.

Os principais achados de necropsia foram coloração vermelha intensa da mucosa do intestino delgado e edema 
da parede da vesícula biliar. As mais importantes alterações histológicas foram degeneração hidrópico-vacuolar do epitélio tubular renal em três dos cinco bovinos que morreram.

TERMOS DE INDEXAÇÃO: Plantas tóxicas, intoxicação por planta, Mascagnia sp, patologia, bovinos, "morte súbita".

\section{INTRODUÇÃO}

No Brasil é muito frequente a ocorrência de "morte súbita" nos bovinos, causada por ingestão de plantas tóxicas. Essas mortes repentinas em geral se manifestam sem sinais clínicos prévios e ausência, na necropsia, de achados macroscópicos de significado. Ao exame histológico, porém, em muitos animais intoxicados encontra-se no rim lesão característica no epitélio dos túbulos contornados distais, uma degeneração hidrópico-vacuolar acompanhada de cariopicnose. Foram descritas, até o momento, 11 plantas capazes de provocar esse quadro e esse tipo de lesão no Brasil. A principal é Palicourea marcgravii, da família Rubiaceae, planta de larga distribuição, responsável pela grande maioria das mortes de bovinos no Brasil (Tokarnia \& Döbereiner 1986). Outras plantas do gênero capazes de causar mortes súbitas tem distribuição geográfica bem mais limitada. Assim há Palicourea juruana, nos Estados do Pará, Amazonas e Rôndonia (Tokarnia \& Döbereiner 1982), Palicourea grandiflora, nos Estados de Rondônia, Acre e Mato Grosso (Tokarnia et al.1981), Palicourea aeneofusca em Pernambuco e na Bahia (Tokarnia et al. 1983). No gênero Mascagnia, as plantas capazes de determinar "morte súbita" são Mascagnia rigida nas regiões Nordeste e Sudeste (Tokarnia et al. 1961, Santos 1975, Tokarnia et al. 1994), Mascagnia pubiflora, na parte sul da Região Centro-Oeste, estendendo-se para dentro do Estado de São Paulo (Fernandes \& Macruz 1964, Tokarnia \& Döbereiner 1973), Mascagnia elegans, no Estado de Pernambuco (Couceiro et al.1976) e Mascagnia aff. rigida, na parte setentrional do Estado do Espirito Santo (Tokarnia et al. 1985). Entre as bignoniáceas há Pseudocalymma elegans, no Estado do Rio de Janeiro ( Mello \& Fernandes 1941, Tokarnia et al. 1969), Arrabidaea bilabiata na Bacia Amazônica (Döbereiner et al. 1983) e Arrabidaea japurensis no Estado de Roraima (Tokarnia \& Döbereiner 1981).

No caso da doença que ocorre na região Litorânea de Santa Catarina, os primeiros históricos sobre mortes súbitas foram obtidos em 1992, no município de Tubarão. Um criador de bovinos informou que, em sua propriedade, sempre que se movimentava o gado para vacinações ou vermifugações, alguns animais caíam e morriam em questão de minutos. Partindo-se dessas informações, foi efetuado um levantamento epidemiológico e foram realizados experimentos em bovinos com plantas suspeitas de causar essas mortes.

\section{MATERIAL E MÉTODOS}

Foram coletados históricos sobre a ocorrência de "mortes súbitas" em bovinos no Litoral do Estado de Santa Catarina. Amostras de vísceras de três bovinos com histórico de "morte súbita" foram avaliados através de exames histológicos.
Três plantas suspeitas foram coletadas e administradas a bovinos em dose única de $20 \mathrm{~g} / \mathrm{kg}$. Em decorrência dos resultados obtidos, a trepadeira Mascagnia sp (Fig. 1) foi submetida a experimentos adicionais. Folhas verdes desta planta foram coletadas, conservadas em geladeira e administradas por via oral a nove bovinos, em doses únicas crescentes (Quadro 1).

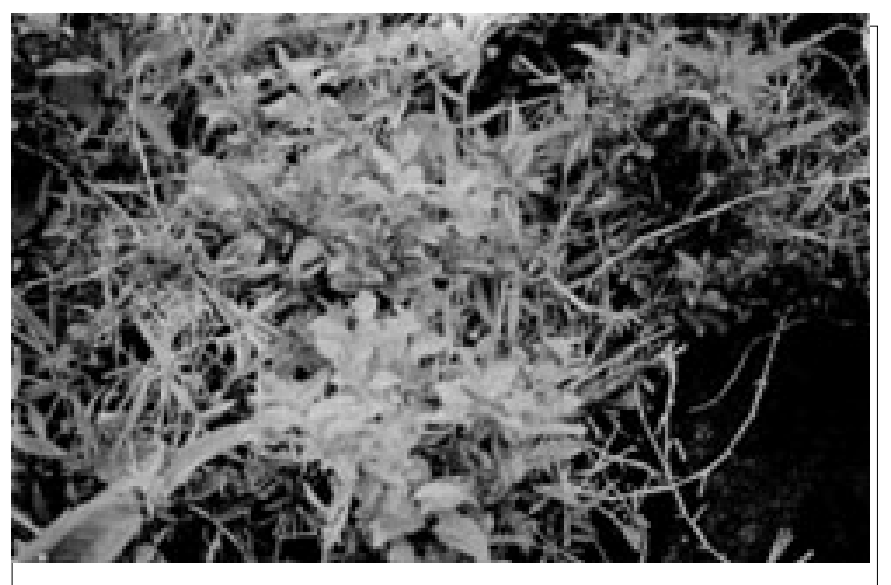

Fig. 1. Mascagnia sp, município de Tubarão, Santa Catarina.

Os animais em experimentação eram mantidos estabulados durante a noite, e eram soltos durante o dia em piquetes com "quicuio" (Penisetum clandestinum) e água a vontade. Antes e após a administração da planta os animais foram examinados e, 14 a 23 horas após a ingestão da planta, foram movimentados por um período de 5 a 25 minutos. Os experimentos foram realizados nas dependências do Laboratório de Patologia Animal, Centro de Ciências Agroveterinárias em Lages, Santa Catarina. Os animais que morreram foram necropsiados, coletando-se amostras de pulmão, coração, fígado, rins, linfonodos, tubo digestivo, sistema nervoso central e músculos. Estas foram fixadas em formol a $10 \%$, incluídas em parafina, cortadas a 5 micras e coradas por hematoxilina-eosina. Fragmentos de rim foram cortados por congelamento e corados por Sudan III.

\section{RESULTADOS}

Intoxicação natural

Dados epidemiológicos evidenciaram que as mortes súbitas são frequentes em bovinos nos municípios de Tubarão, Imaruí, Jaguaruna, São Ludgero, Braço do Norte, Pedras Grandes, Urussanga, Nova Veneza e Jacinto Machado, no Estado de Santa Catarina. A maioria dos animais era encontrada morta, sem terem sido observadas prévias manifestações clínicas, ou os animais morriam em questão de minutos após serem movimentados. Alguns bovinos apresentavam apatia, anorexia, andar rígido, ficando a maior parte do tempo deitados e morriam, ou se recuperavam em 3 a 4 dias.

A região é, em sua maior parte, constituída de minifúndios, onde o número de bovinos para cada propriedade gira entre 10 e 20 cabeças. Alguns proprietários chegaram a perder por morte repentina até $40 \%$ do rebanho, em apenas um ano. A maioria dos criadores informou que a doença ocorria há muitos anos e que a causa deste tipo de morte sempre era atribuída ao carbúnculo hemático ou à intoxicação por "timbó", um cipó leitoso. 
Quadro 1. Experimentos em bovinos com as folhas verdes de Mascagnia sp

\begin{tabular}{|c|c|c|c|c|c|c|c|}
\hline $\begin{array}{l}\text { Bovino } \\
n^{\circ} \text { (SAP- } \\
\text { UDESC) }\end{array}$ & $\begin{array}{c}\text { Peso } \\
\mathrm{kg}\end{array}$ & $\begin{array}{l}\text { Dose } \\
\mathrm{g} / \mathrm{kg}\end{array}$ & $\begin{array}{c}\text { Ínicio dos } \\
\text { sinais clínicos } \\
\text { após administração }\end{array}$ & $\begin{array}{l}\text { Principais manifestações } \\
\text { clínicas }\end{array}$ & $\begin{array}{l}\text { Morte ou } \\
\text { recuperação após } \\
\text { administração }\end{array}$ & $\begin{array}{l}\text { Alterações } \\
\text { macroscópicas }\end{array}$ & $\begin{array}{l}\text { Alterações } \\
\text { microscópicas }\end{array}$ \\
\hline 61 & 120 & 3 & - & Ausência de sintomas & & - & \\
\hline 60 & 190 & 5 & $14 \mathrm{~h} 50 \mathrm{~min}$ & $\begin{array}{l}\text { Ao ser movimentado manifestava leves tremores muscu- } \\
\text { lares, contrações bruscas, taquicardia, deitando-se rapi- } \\
\text { damente }\end{array}$ & $96 \mathrm{~h}$ & - & - \\
\hline 66 & 91 & 5 & $23 \mathrm{~h}$ & $\begin{array}{l}\text { Durante a movimentação manifestou cansaço, ficando de } \\
\text { pé com a cabeça baixa }\end{array}$ & $37 \mathrm{~h} 40 \mathrm{~min}$ & - & - \\
\hline 67 & 130 & 7,5 & $12 \mathrm{~h}$ & $\begin{array}{l}\text { Sempre que movimentado manifestava cansaço, com tre- } \\
\text { mores musculares leves, deitando-se rapidamente, jugular } \\
\text { tensa, taquicardia e orelhas caídas }\end{array}$ & $72 \mathrm{~h}$ & - & - \\
\hline $\begin{array}{c}69 \\
(5269)\end{array}$ & 145 & 7,5 & $12 \mathrm{~h} 35 \mathrm{~min}$ & $\begin{array}{l}\text { Estava pastando normalmente. Apenas manifestava } \\
\text { taquicardia. Uma hora após foi encontrado morto }\end{array}$ & $13 \mathrm{~h} 35 \mathrm{~min}$ & $\begin{array}{l}\text { Conteúdo liquido avermelhado no } \\
\text { jejuno e íleo, com acentuada co- } \\
\text { loração vermelha da mucosa }\end{array}$ & Sem alterações \\
\hline $\begin{array}{c}64 \\
(4337)\end{array}$ & 65 & 10 & $13 \mathrm{~h} 20 \mathrm{~min}$ & $\begin{array}{l}\text { Taquicardia. Foi movimentado por } 25 \text { minutos. Repenti- } \\
\text { namente manifestou tremores musculares, respiração ofe- } \\
\text { gante, caindo lateralmente, com contrações bruscas dos } \\
\text { membros, jugular tensa, momento de calma e morte }\end{array}$ & $14 \mathrm{~h}$ & $\begin{array}{l}\text { Petéquias no epicárdio. Coloração } \\
\text { vermelha da mucosa do intesti- } \\
\text { no delgado sendo mais evidente } \\
\text { no jejuno e íleo e edema sub-se- } \\
\text { roso na vesícula biliar }\end{array}$ & $\begin{array}{l}\text { Degeneração hidrópico-vacuolar em } \\
\text { alguns tú-bulos uriníferos contorna- } \\
\text { dos distais, congestão e hemorragias } \\
\text { moderadas na mucosa do intestino } \\
\text { delgado com dilatação de vasos lin- } \\
\text { fáticos e edema intrafolicular }\end{array}$ \\
\hline $\begin{array}{c}65 \\
(4333)\end{array}$ & 116 & 10 & $13 \mathrm{~h} 15 \mathrm{~min}$ & $\begin{array}{l}\text { Taquicardia com batimentos fortes. Movimentado por } 15 \\
\text { minutos, repentinamente teve taquipnéia, deitando-se ra- } \\
\text { pidamente, jugular tensa, contrações dos membros, mo- } \\
\text { mento de calma e morte }\end{array}$ & 14h $30 \mathrm{~min}$ & $\begin{array}{l}\text { Coloração vermelha acentuada da } \\
\text { mucosa do intestino delgado e } \\
\text { edema subseroso na vesícula bi- } \\
\text { liar e nos linfonodos mediastinais }\end{array}$ & $\begin{array}{l}\text { Degeneração hidrópico-vacuolar de } \\
\text { túbulos uriníferos contornados dis- } \\
\text { tais; no coração macrófagos ao redor } \\
\text { das coronárias e de fibras de Purkin- } \\
\text { je; congestão, hemorragias e dilata- } \\
\text { ção linfática na mucosa do intestino } \\
\text { delgado e eosinofilia de miofribras }\end{array}$ \\
\hline $\begin{array}{c}62 \\
(4298)\end{array}$ & 130 & 15 & $11 \mathrm{~h} 45 \mathrm{~min}$ & $\begin{array}{l}\text { Apatia, taquicardia, edema submandibular, andar lento } \\
\text { frustrando a tentativa de movimentação, defecação difí- } \\
\text { cil. Encontrado morto }\end{array}$ & $19 \mathrm{~h} 30 \mathrm{~min}$ & $\begin{array}{l}\text { Edema subcutâneo, ascite e } \\
\text { edema no mesentério }\end{array}$ & $\begin{array}{l}\text { Degeneração hidrópico-vacuolar nos } \\
\text { túbulos uriníferos contornados dis- } \\
\text { tais, congestão hepática centrolo- } \\
\text { bular e eosinofilia de miofribras }\end{array}$ \\
\hline $\begin{array}{c}68 \\
(4438)\end{array}$ & 78 & 20 & $19 \mathrm{~h}$ & $\begin{array}{l}\text { Fezes secas com muco, apatia, anorexia, decúbito. Encon- } \\
\text { trado morto na baia. }\end{array}$ & $40 \mathrm{~h}$ & $\begin{array}{l}\text { Fígado com aspecto de noz-mos- } \\
\text { cada, edema da vesícula biliar, } \\
\text { ressecamento do conteúdo do } \\
\text { omaso e do intestino grosso }\end{array}$ & $\begin{array}{l}\text { Eosinofilia de fibras cardíacas, macró- } \\
\text { fagos circundando as coronárias, e no } \\
\text { figado congestão centro lobular com } \\
\text { tumefação e necrose difusa dos hepa- } \\
\text { tócitos }\end{array}$ \\
\hline
\end{tabular}


Das amostras de vísceras de três bovinos com histórico de "morte súbita", enviadas ao Laboratório de Patologia Animal para exames histológicos, em uma foi constatada degeneração hidrópico-vacuolar nas células epiteliais dos túbulos uriníferos contornados distais.

\section{Intoxicação experimental}

Os dados mais importantes dos experimentos da administração de doses únicas das folhas frescas de Mascagnia sp constam do Quadro 1.

$\mathrm{O}$ bovino que recebeu dose única das folhas frescas de 3 $\mathrm{g} / \mathrm{kg}$ não adoeceu. Os seis bovinos que receberam doses únicas de $5 \mathrm{~g} / \mathrm{kg}, 7,5 \mathrm{~g} / \mathrm{kg}$ e $10 \mathrm{~g} / \mathrm{kg}$, apresentaram, quando movimentados, manifestações clínicas de cansaço, jugular ingurgitada, leves tremores musculares e, às vezes, contrações bruscas. Taquicardia com movimentos fortes era detectada antes da movimentação e se acentuava rapidamente após exercício. Quatro dos seis bovinos deste grupo após estas manifestações deitaram ou caíram de lado subitamente (Bov. 60: $5 \mathrm{~g} / \mathrm{kg}$, Bov. 67: 7,5 g/kg, Bov. 64 e 65: $10 \mathrm{~g} / \mathrm{kg}$ ). Destes, dois se recuperaram (Bov. 60: $5 \mathrm{~g} / \mathrm{kg}$, Bov. 67: 7,5 g/ kg); os outros dois morreram (Bov. 64 e 65: $10 \mathrm{~g} / \mathrm{kg}$ ), tendo passado desde o início da apresentação de sintomas até a morte 40 minutos e $\mathrm{lh} 15 \mathrm{~min}$, respectivamente. Dos outros dois bovinos deste grupo de seis, que adoeceram mas não se deitaram ou cairam subitamente, um se recuperou (Bov. 66: $5 \mathrm{~g} / \mathrm{kg}$ ) e o outro foi encontrado morto 1 hora após o início da manifestação dos sintomas (Bov.69: 7,5 g/kg).

Não foi possível movimentar devidamente os dois bovinos que receberam doses únicas de 15 e $20 \mathrm{~g} / \mathrm{kg}$ (Bov. 62: 15 $\mathrm{g} / \mathrm{kg}$, Bov. 68: $20 \mathrm{~g} / \mathrm{kg}$ ). Estes animais mostraram andar lerdo, apatia, permanecendo a maior parte do tempo deitados evitando qualquer movimentação; foram encontrados mortos respectivamente $7 \mathrm{~h} 45 \mathrm{~min}$ e 21 horas após terem sido observados os primeiros sinais clínicos.
À necropsia as principais lesões consistiram de coloração vermelha intensa da mucosa do intestino delgado (Bov.69, 64 e 63), sendo mais acentuada no jejuno e íleo, e edema na subserosa da parede da vesícula biliar (Bov. 64,65 e 68). Fígado com aspecto de noz-moscada foi observado em um animal (Bov. 68); edema gelatinoso leve nas regiões sub-mandibular e esternal, e acentuado no mesentério, foi visto em outro animal (Bov. 62).

Pelos exames histológicos foi verificado no rim degeneração hidrópico-vacuolar do epitélio dos tubulos contornados distais (Bov. 62, 64 e 65) (Fig. 2); no coração, eosinofilia de fibras musculares com pequeno acúmulo de macrófagos principalmente ao redor de vasos, e das fibras de Purkinje (Bov. 62, 65 e 68); e no fígado, congestão centrolobular (Bov. 62 e 68) com tumefação e necrose difusa dos hepatócitos (Bov. 68). Em todos os bovinos, exceto um (Bov. 68), havia congestão e hemorragias na mucosa do intestino delgado, associadas a dilatacão acentuada dos capilares linfáticos das vilosidades, e separação por edema dos elementos celulares dos folículos linfóides.

\section{DISCUSSÃO E CONLUSÕES}

Experimentalmente se demonstrou que $5 \mathrm{~g} / \mathrm{kg}$ das folhas frescas de Mascagnia sp, foi a menor dose única capaz de provocar o aparecimento de sinais clínicos de intoxicação, quando os animais eram submetidos a movimentação, sem contudo causar morte. Dos dois bovinos que receberam $7,5 \mathrm{~g} / \mathrm{kg}$ da planta, um morreu. Doses únicas de $10 \mathrm{~g} / \mathrm{kg}$ causaram a morte dos dois bovinos. Os três bovinos tiveram "morte súbita" com ou sem sinais clínicos prévios. A morte ocorreu entre 13 e 15 horas após a administração da planta.

Os bovinos que ingeriram doses superiores a $15 \mathrm{~g} / \mathrm{kg}$ manifestaram sinais clínicos prévios bem definidos, porém, o tempo decorrido da ingestão à morte foi maior do que nos

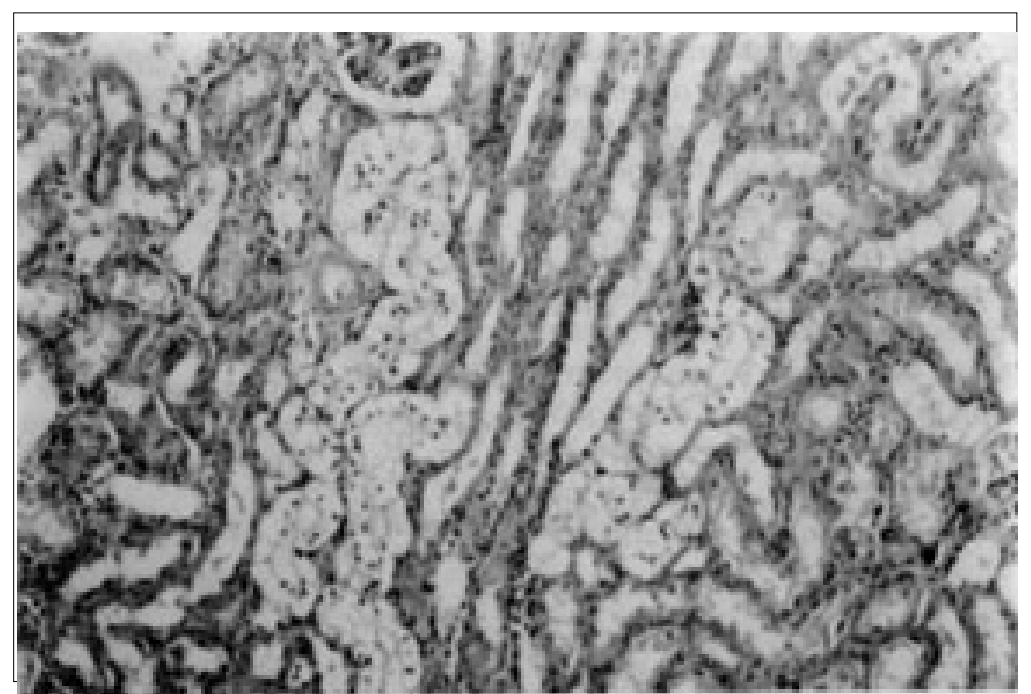

Fig. 2. Degeneração hidrópico-vacuolar do epitélio nos túbulos uriníferos contornados distais, na intoxicação experimental por Mascagnia sp (Bovino 64). HE, obj.25. 
animais que ingeriram doses menores. Isto pode ser explicado pelo fato de que esses animais permaneceram deitados e evitavam qualquer movimentação. Diante disto, parece ser a movimentação um fator importante na manifestação da "morte súbita".

Alguns achados clínico-patológicos, como o ingurgitamento da jugular, os edemas e a congetão hepática (em um caso o fígado apresentando aspecto de noz-moscada), e possivelmente a congestão da mucosa intestinal, podem ser atribuídos à ação direta da planta sobre o coração, causando uma insuficiência cardíaca aguda.

Analisando-se o quadro clínico-patológico da doença natural com histórico de "morte súbita" e comparando-o com os resultados obtidos experimentalmente em bovinos, através da administração de folhas frescas de Mascagnia sp, bem como a presença dessa planta em todas as propriedades onde ocorria a doença, pode-se concluir que esta planta é a responsável pelas mortes repentinas que ocorrem no Litoral de Santa Catarina.

A suspeita de criadores e veterinários, que os bovinos encontrados mortos sem prévia manifestação clínica, seriam vítimas do carbúnculo hemático, deve ser descartada pela ausência das lesões e das características epidemiológicas desta doença. Da mesma forma, a suspeita de intoxicação pelos cipós leiteiros conhecidos por "timbós" (Oxypetalum spp), não deve ser considerada, uma vez que estas plantas não se revelaram tóxicas para bovinos (Tokarnia \& Gava, dados não publicados).

Na mata os ramos de Mascagnia sp se estendem sobre a copa das árvores, ficando fora do alcance do gado. Os bovinos ingerem as folhas dessa trepadeira quando esta é encontrada em capoeiras, onde a vegetação é baixa e em locais de desmatamento recente, onde ocorre muita brotação da planta, ou quando ocorre a queda de sementes com o nascimento de grande número de plantas.

\section{REFERÊNCIAS}

Couceiro J.E.M., Silva A.C.C. \& Silva J.A.1976. Observações e ensaios sobre a alegada intoxicação de bovinos por plantas, no Estado de Pernambuco. Resumo em Anais XV Congr. Bras. Med. Vet., Rio de Janeiro, e Relatório.

Döbereiner J., Tokarnia C.H. \& Silva M.F. 1983. Intoxicação por Arrabidaea bilabiata (Bignoniaceae) em bovinos na Região Amazônica do Brasil. Pesq. Vet. Bras. 3(1):17-24.

Fernandes N.S. \& Macruz R.1964. Toxicidade da “corona” - Mascagnia pubiflora (Juss.) Griseb. (Malpighiaceae). Arqs Inst. Biológico, São Paulo, 31(1):1-4.

Mello E.M.M. \& Fernandes J.S. 1941. Contribuição ao estudo de plantas tóxicas brasileiras. Serv. Inf. Agrícola, Min. Agricultura, Rio de Janeiro.

Santos H.L. 1975. Aspectos clínicos, laboratoriais e anatomo-histopatológicos na intoxicação experimental de bovinos pela Mascagnia rigida $\mathrm{Gr}$. Tese de Mestrado, Esc. Vet. UFMG, Belo Horizonte. 36 p. (Resumo em Arqs Esc. Vet., UFMG, Belo Horizonte, 27(3):398-399.)

Tokarnia C.H., Döbereiner J. \& Peixoto P. V. 1994. Aspectos clínico-patológicos complementares da intoxicação por algumas plantas tóxicas brasileiras. Pesq. Vet. Bras. 14(4):111-122.

Tokarnia C.H., Canella C.F.C., \& Döbereiner J. 1961. Intoxicação por um “tingui”" (Mascagnia rigida Griseb.) em bovinos no Nordeste do Brasil. Arqs Inst. Biol. Anim., Rio de J., 4:203-215.

Tokarnia C.H., Döbereiner J., Canella C.F.C. \& Guimarães D.J. 1969. Intoxicação experimental por Pseudocalymma elegans (Vell.) Kuhlm. em bovinos. Pesq. Agropec. Bras. 4:195-204.

Tokarnia C.H. \& Döbereiner J. 1973. Intoxicação por Mascagnia pubiflora em bovinos no Estado de Mato Grosso. Pesq. Agropec. Bras., Sér. Vet. 8:61-68.

Tokarnia C.H. \& Döbereiner J. 198. Intoxicação por Arrabidaea japurensis (Bignoniaceae) em bovinos em Roraima. Pesq. Vet. Bras. 1:7-17.

Tokarnia C.H., Döbereiner J. \& Silva M.F. 1981. Intoxicação por Palicourea grandiflora (Rubiaceae) em bovinos no Território de Rondônia. Pesq. Vet. Bras. 1(3):85-94.

Tokarnia C.H. \& Döbereiner J. 1982. Intoxicação experimental por Palicourea juruana (Rubiaceae) em bovinos e coelhos. Pesq. Vet. Bras. 2(1):17-26.

Tokarnia C.H., Döbereiner J., Couceiro J.E.M. \& Silva A.C.C. 1983. Intoxicação por Palicourea aeneofusca (Rubiaceae), a causa de "mortes súbitas" em bovinos na Zona da Mata de Pernambuco. Pesq. Vet. Bras. 3(3):75-79.

Tokarnia C.H., Döbereiner J. \& Peixoto P.V. 1985. Intoxicação por Mascagnia aff. rigida (Malpighiaceae) em bovinos no Norte do Espirito Santo. Pesq. Vet. Bras. 5(3):77-91.

Tokarnia C.H. \& Döbereiner J. 1986. Intoxicação por Palicourea marcgravii (Rubiaceae) em bovinos no Brasil. Pesq. Vet. Bras. 6(3):73-92. 